\title{
Learning about Stellar Dynamos from Long-term Photometry of Starspots
}

\author{
Douglas S. Hall \\ Center of Excellence Tenessee State University Nashville, Tennessee \\ 37203
}

\begin{abstract}
Spottedness, as evidenced by photometric variability in 277 late-type binary and single stars, is found to occur when the Rossby number is less than about $2 / 3$. This holds true when the convective turnover time versus $B-V$ relation of Gilliland is used for dwarfs and also for subgiants and giants if their turnover times are twice and four times longer, respectively, than for dwarfs. Differential rotation is found correlated with rotation period (rapidly rotating stars approaching solid-body rotation) and also with lobe-filling factor (the differential rotation coefficient $k$ is 2.5 times larger for $F=0$ than $F=1)$. Also reviewed are latitude extent of spottedness, latitude drift during a solartype cycle, sector structure and preferential longitudes, starspot lifetimes, and the many observational manifestations of magnetic cycles.
\end{abstract}

\section{Introduction}

This will be a review of photometry of stars which are variable as a result of starspot activity, and how these observations can be useful in providing verification of various dynamo theories.

There is much other observational material which has been useful also (like spectroscopy of chromospheric emission, far ultraviolet emission from the transition region, $\mathrm{x}$-ray emission from the corona, radio emission, flares, and direct measures of the magnetic field) but I have the impression that starspots have been somewhat neglected or, at least, not used to their full potential.

One emphasis will be on the continuous long-term behavior of starspots. Much of the existing work has provided only individual snapshots of spotted stars taken once or at random times.

My second emphasis will be to include all of the types of stars in which stellar dynamos may be operating. Often comparison between theory and observation will consider only one type of star, for example, only main-sequence dwarfs or only RS 
CVn-type binaries. A wide net is important because it can explore wide ranges of possibly important factors:

rotation periods from less than 2 hours to longer than a year

spectral types from $\mathrm{F}$ to $\mathrm{K}$

dwarfs to giants

strong tidal interaction (contact binaries) to weak (single stars)

shallow convection to deep convection

time scales from minutes (flares) to centuries (magnetic cycles)

Table 1 is a list of the 11 types of stars, separately defined, which show one or more aspects of chromospheric activity, i.e., solar-type activity probably related in some way to dynamo action. For references to each type, see Hall (1987), Strassmeier et al. (1988), Hall (1989), and Hall (1990b). Note that in some types the active star is single, in some types it occurs in a binary, and some types include single stars and binaries.

\section{Spottedness as a function of Rossby number}

The most outstanding observational manifestation of starspots is the so-called wave. Numerous examples of waves are seen in Strassmeier and Hall (1988, figures $1-5)$ and in Strassmeier et al. (1989, figures 1-49). These are light curves of 54 chromospherically active stars, taken over a four-year interval with an automatic telescope in Arizona. The vertical axis is magnitude in $V$; the horizontal axis is Julian date. The brightness in the continuum varies as the star rotates because one hemisphere is much darker than the other as a result of a concentration of dark starspots distributed unevenly in stellar longitude. The amplitudes are often as large as $0 .{ }^{m} 2$, which is about $20 \%$ and would be produced by a dark region about $20^{\circ}$ in radius. The largest wave amplitude yet seen, $0 .^{m} 5$ in the $V$ band, was in the late-1986 light curve of II Peg (Doyle et al., 1988).

Previous attempts have been made to demonstrate a functional dependence between wave amplitude, or spottedness, and some parameter such as rotation period, spectral type, or $H \& K$ flux. Examples are Catalano et al. (1980, figure 4 ) and Strassmeier (1989, figure 4). To my knowledge, none has succeeded. The problem, I think, is that the samples studied were restricted to stars already known to be heavily spotted and did not probe extremes in parameter space.

Therefore I have searched many sources for stars of many different types which have a measured wave amplitude, even those demonstrated to vary less than 1 $\%$, i.e., a wave amplitude less than $0 .^{m} 01$. Altogether 277 such stars were found. Table 2 lists my principal sources. For binaries, in which only one of the two stars is spotted, the observed amplitude has been corrected for the dilution caused by the other star's light. For non-variable stars, the rotation period was estimated from the observed $v \sin i$ and the canonical radius for the corresponding luminosity class. One result is shown in the first three figures.

Figure 1 shows the dwarfs, i.e, main-sequence stars. The vertical axis is log of the rotation period. The horizontal axis is $B-V$. The open circles indicate 
Table 1. Types of stars with choromospheric activity

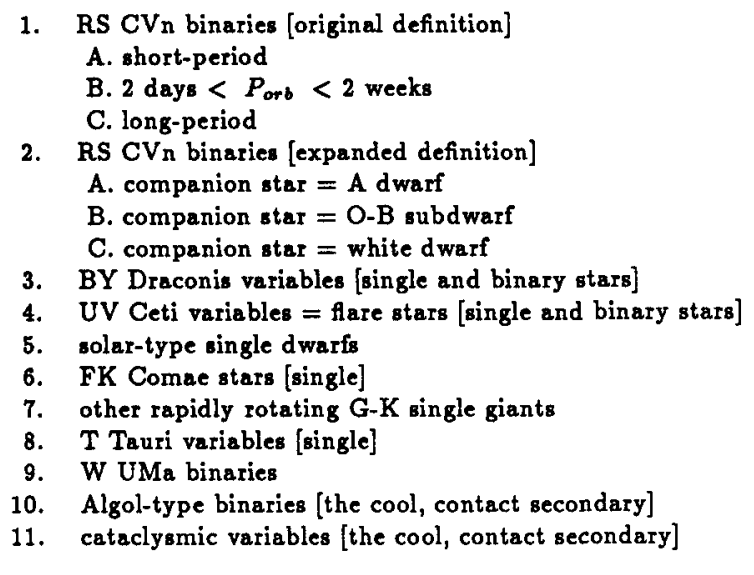

Table 2. F-G-K stars searched for variability

1. eclipsing F-type and G-type main-sequence binaries, [Publ. Univ. Pennaylvania Astr. Ser. XII]

2. W UMa binaries, [I.A.U. Collog. 29, 287]

3. Algol-type binaries, [1.A.U. Colloq. 107, 219]

4. T Tauri stars, [Publ. Aotron. Soc. Pac. 88, 1088]

5. UBV standards, [Astrophys. J. 117, 313]

6. $G$ and $K$ dwarfs in the O.C. Wilson $H$ \& $K$ emission survey, [Astrophys. J. 279, 763]

7. Lowell Observatory photometry of the Wilson survey stars,

[Lockwood and Skiff AFGL-TR-88-0221]

8. Arizona-Tonantzintla Catalogue (the standards), [Comm. Lunar and Planetary Lab. No. 63]

9. Catalogue of Chromospherically Active Binary Stars (plus candidates)

[Astron. Astrophys. Suppl. Ser. 72, 291]

10. four years of photometry with an automatic telescope

[A strophys. J. Suppl. Ser. 67, 439 - the constant stars]

[Astrophys. J. Suppl. Ser. 67, 453 - the single stars]

[Astrophys. J. Suppl. Ser. 69, 141 - the binary stars]

11. Photometry of 50 Suspected Chromopspherically Active Variables

[Astrophys. J. Suppl. Ser. 74, 225]

12. Thirteen Chromospherically Active Ellipsoidal Variables, [Astron. J. 100, 554]

13. single BY Dra variables, [Astron. J. 82, 490]

constant stars, wave amplitudes less than $1 \%$. The plusses indicate stars with wave amplitudes of $1 \%$ or greater. The solid line is the convective turnover time taken from Gilliland (1985, figure 10) for main-sequence stars. Any star above this curve should have a Rossby number greater than unity, on the curve a Rossby number of unity, and below the curve a Rossby number less than unity. It is demonstrated very nicely, I think, that a Rossby number a little less than unity is a sharp dividing line between the non-variable and the variable stars.

Figure 2 is the same thing for the subgiants, i.e, luminosity class IV. The curve is the same convective turnover times for $d$ warfs, but we see now that the dividing 


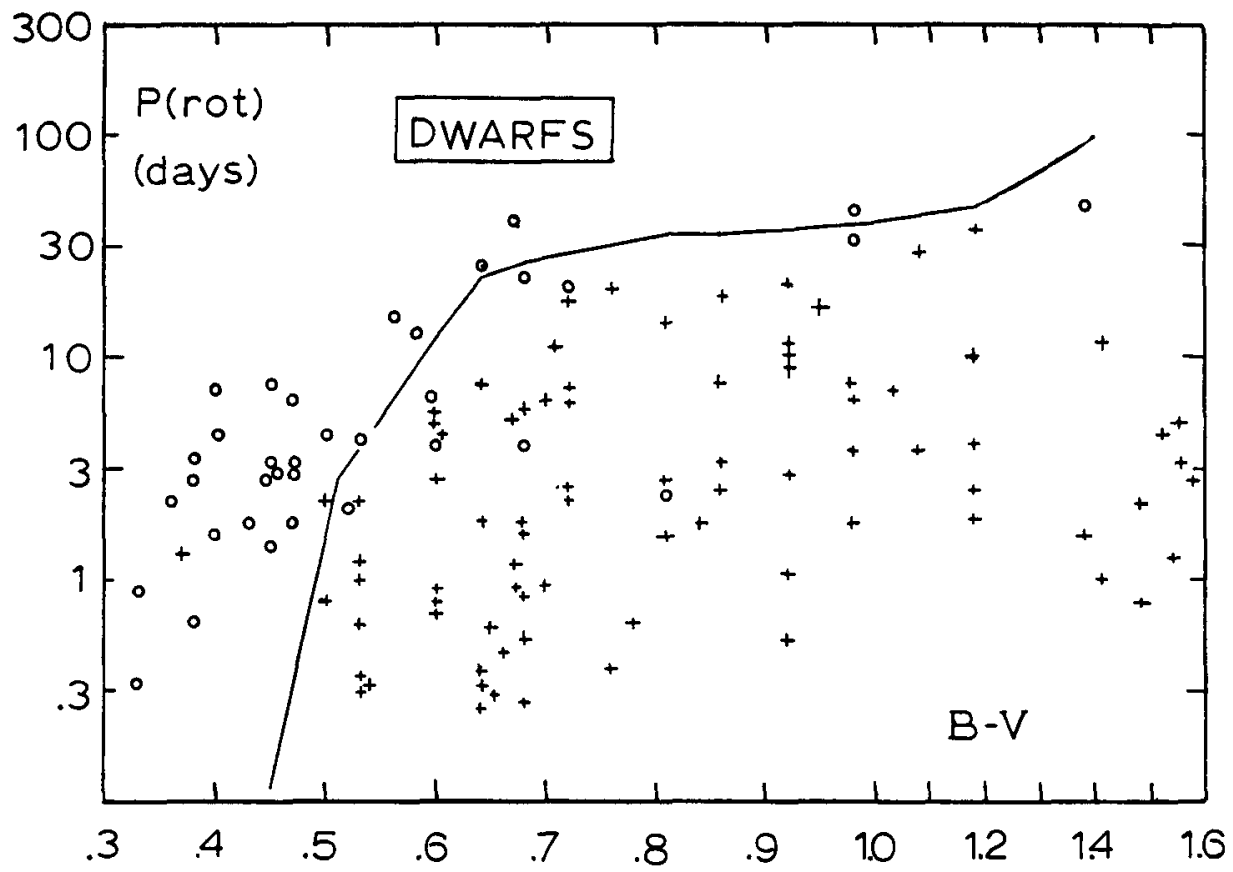

Fig. 1. $P_{\text {rot }}$ versus $B-V$ for dwarfs. Variables $(+)$ and non-variables $(\bigcirc)$ are distinguished. The solid curve represents Gilliland's convective turnover times.

line between non-variable and variable lies somewhat higher - about 0.3 in the $\log$ or turnover times longer by a factor 2 compared to dwarfs of the same $B-V$.

Figure 3 is the same thing for the giants, i.e., luminosity class III. We see here that the dividing line between non-variable and variable lies even higher - about 0.6 in the log or turnover times longer by a factor 4 compared to dwarfs of the same $B-V$.

Gilliland's turnover times show that, as a star evolves to the base of the giant branch (this would be the subgiants), his turnover times become longer by about 0.5 in the log. So my results for the subgiants are in fair accordance with his. But as a star evolves up the giant branch his turnover times become shorter. For a typical late-type star of luminosity class III they would be shorter than the mainsequence times by about 0.6 in the $\log$, whereas mine were longer by about 0.6 in the log. So my results for the giants are not in accordance with his.

For the next three figures I have computed Rossby numbers for all of my 277 stars, using Gilliland's convective turnover times for the dwarfs but longer turnover times for the subgiants and the giants. The amounts added to the log of the mainsequence turnover times were 


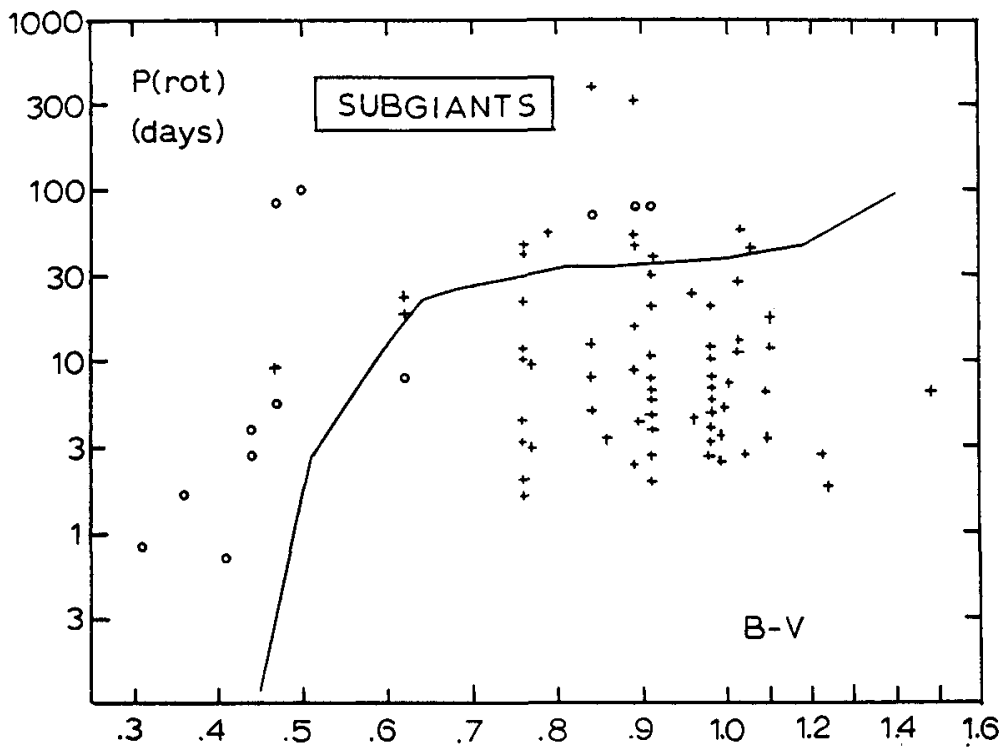

Fig. 2. Same as Figure 1, for subgiants. Note that variability occurs at rotation periods longer than the convective turnover times for dwarfs.

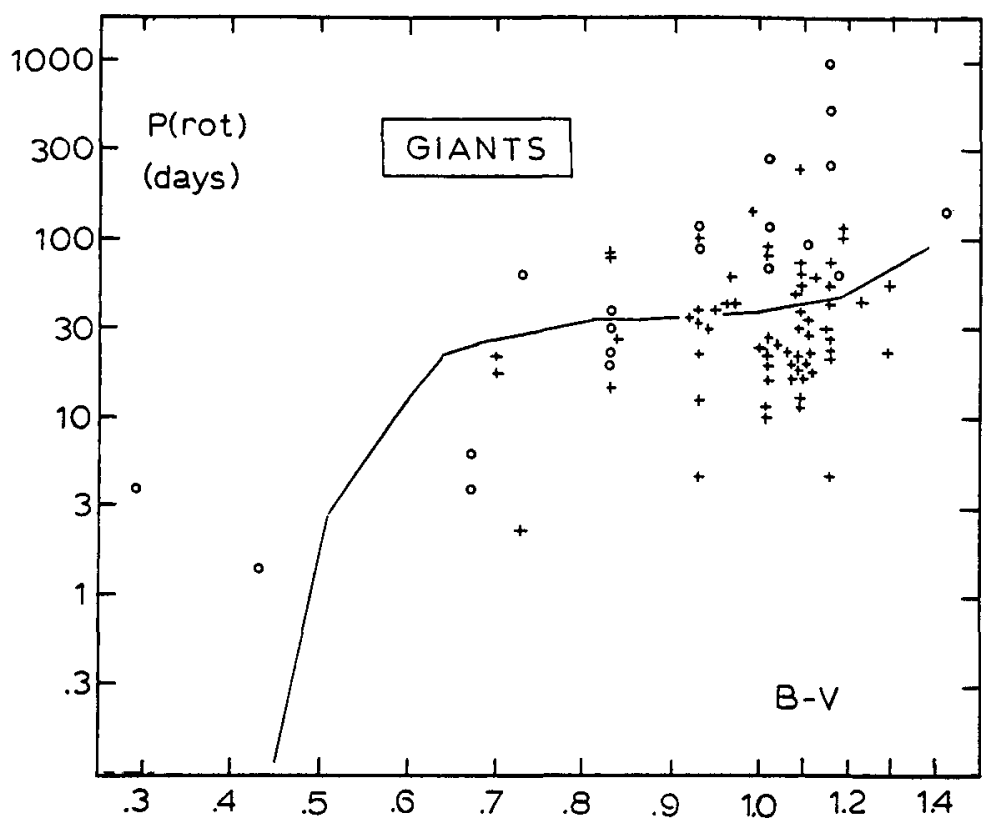

Fig. 3. Same as Figure 1, for giants. Note that variability occurs at rotation periods longer than the turnover times for dwarfs, more so than for the subgiants. 


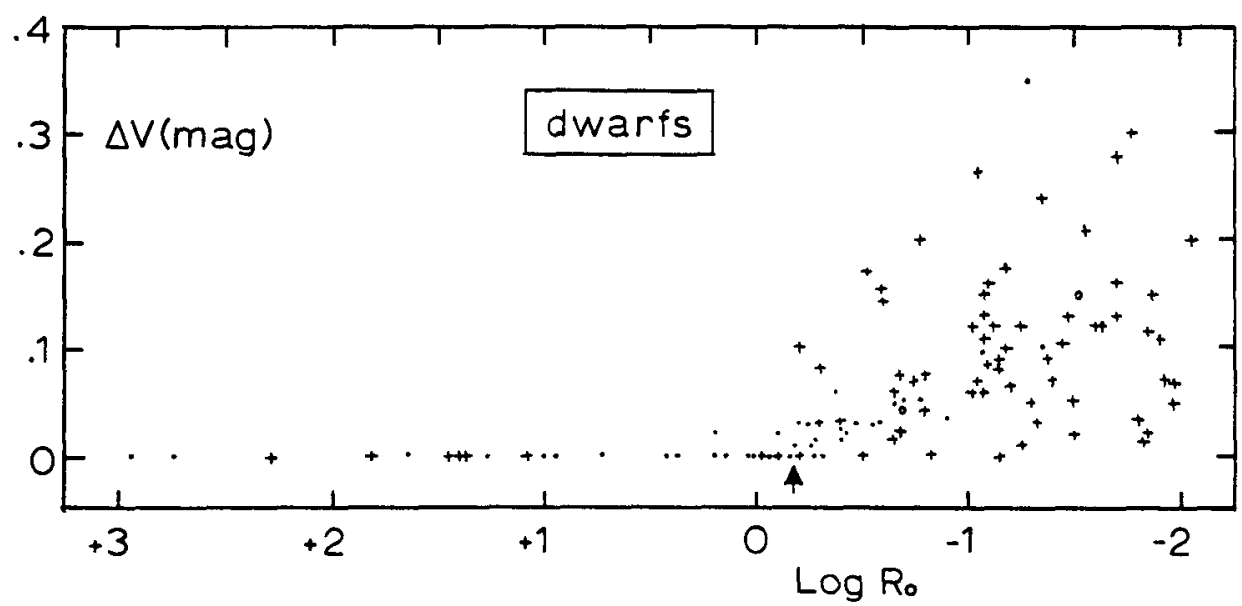

Fig. 4. Starspot wave amplitude versus log Rossby number, for dwarfs. Note the sharp onset of variability at $R o=2 / 3$, marked by the arrow, for both binaries $(+)$ and single stars $(\bullet)$. The Sun is at $\log R o \approx 0$.

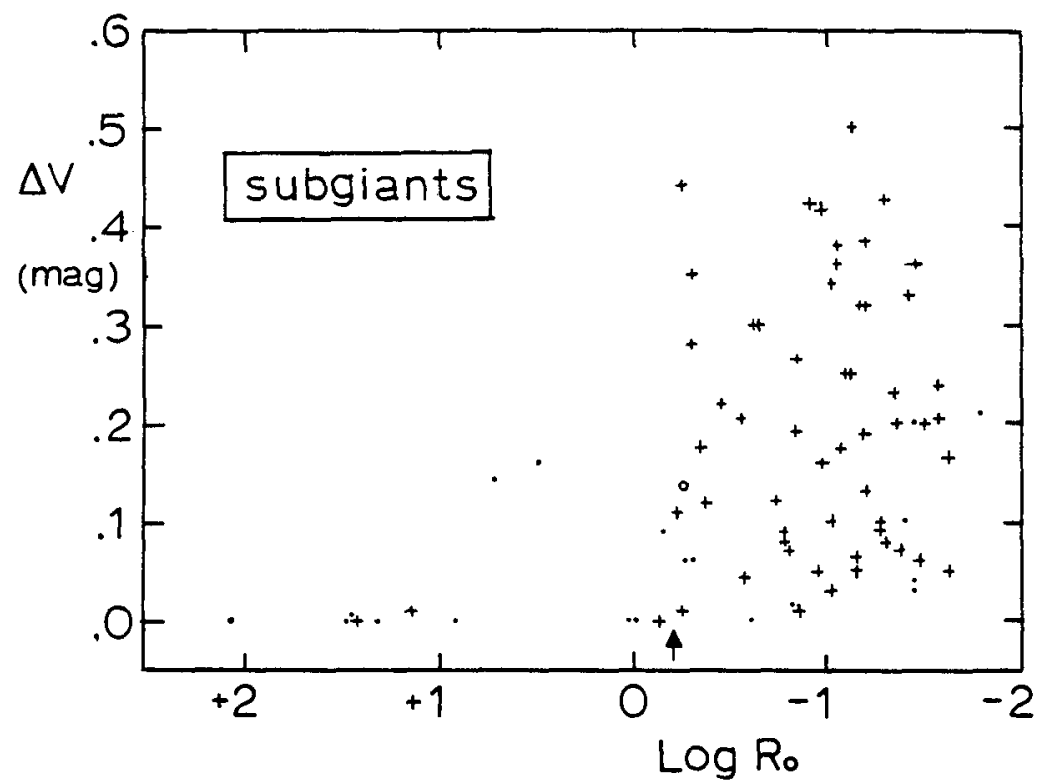

Fig. 5. Same as Figure 4, for subgiants. If HR 1362 and HD 181943 are considered anomalous, we see the same sharp onset of variability at $R_{0}=2 / 3$. 


$\begin{array}{cc}\text { Luminosity Class } & \text { Correction } \\ \text { V } & 0.00 \\ \text { V-IV } & 0.15 \\ \text { IV } & 0.30 \\ \text { IV-III } & 0.45 \\ \text { III } & 0.60 \\ \text { III-II } & 0.75\end{array}$

Figure 4 is for the dwarfs. The horizontal axis is log Rossby number and the vertical axis is wave amplitude in the $V$ bandpass, in magnitudes. Notice the onset of measurable variability at a Rossby number somewhat less than unity, at about $2 / 3$. Moreover, note that the onset is very sharp, within 0.1 in the log, or a factor of only 1.25 . The Sun appears in this figure at a $\log R o \approx 0$; if it happened to rotate in two weeks rather than one month, it would probably vary in brightness by 2 or 3 percent rather than by only $0.1 \%$. There is some indication that wave amplitude increases as Rossby number decreases from $2 / 3$ to $1 / 10$, but that trend is not very well-defined. The plusses are the binaries, the dots are the single stars, and their distributions are indistinguishable.

Figure 5 is for the subgiants. First let me point out two stars which are probably anomalous. They are HR 1362 and HD 181943, both single G8 subgiants with rotation periods longer than a year but nevertheless quite chromospherically active in the usual ways ( $H$ and $K$ emission, $\mathrm{x}$-ray emission, etc.). Their anomalous status has been the subject of a paper by Strassmeier et al. (1990). If we overlook them, then we see again the onset of variability occurs at a Rossby number of about $2 / 3$ and is very sharp, within $\sim 0.1$ in the log. Here, unlike the dwarfs, starspot wave amplitude rises almost immediately and does not continue to increase at smaller Rossby numbers. The amplitudes themselves are somewhat larger than for the dwarfs, up to a half magnitude. But again the distributions of the binaries (plusses) and the single stars (dots) are indistinguishable.

Figure 6 is for the giants. We see the same very sharp rise at $R o \approx 2 / 3$, no continuing increase as Rossby numbers get smaller, the same large amplitudes, again up to a half magnitude, and no difference between the single stars and the binaries.

The smallest Rossby numbers in my sample were about 0.01 but there is no indication that the wave amplitudes were beginning to decrease, i.e., no evidence that extreme dynamo action suppresses spottedness.

The results seen here are consistent with the rotostat model advanced by Gray (1989a) to explain the abrupt decrease in rotational velocity among single giants later than spectral type G0. His model has dynamo action as the mechanism for braking the rapid rotation seen in early-type giants and maintaining slow rotation in late-type giants. (1) The dramatic increase in Gilliland's turnover times (which makes dynamo action effective) and the abrupt braking which Gray sees both occur at the same value of $B-V$. (2) If we take Gray's $\langle V \sin i\rangle=3.02 \mathrm{~km} \mathrm{~s}^{-1}$ for G7 and G8 III stars, employ the factor $\pi / 4$ to get $\langle V\rangle=3.85 \mathrm{~km} \mathrm{~s}^{-1}$, and adopt a canonical value of $12 R_{\odot}$, we get a rotation period of 158 days. The turnover 


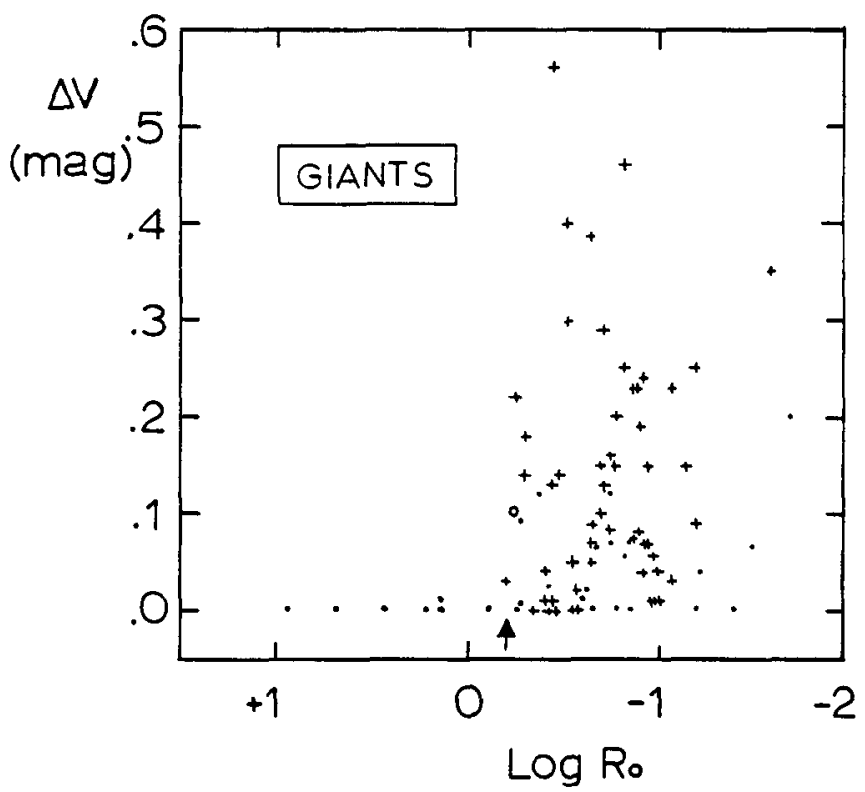

Fig. 6. Same as Figure 4, for giants. The onset of variability at Ro $=2 / 3$ is very sharp and well-defined.

time for a G7.5 III star, based on my previously mentioned adjustment for giants, would be 152 days. The agreement is not quite so good at earlier or later types but still is not bad. At G5 III Gray's $\langle V \sin i\rangle$ leads to a rotation period shorter than my turnover time by a factor 1.5; at K0 III his rotation period is longer, again by a factor 1.5.

\section{Latitude extent}

We all know that sunspots are confined to approximately $30^{\circ}$ above and below the equator. It is now established that large starspot regions can occur at much higher latitudes. In some stars the dark regions form essentially a polar cap. Thus we have an important instance where the solar-stellar analogue breaks down. A tabulation of starspot latitude determinations is given by Gray (1988, table 7-1). Good recent examples are EI Eri, described by Strassmeier (1990) and also discussed by him in a paper at this meeting (see Hackman et al. in these Proceedings), and UX Ari, discussed by Vogt and Hatzes in another paper at this meeting. 
The latitude of a starspot is a relatively difficult parameter to determine, more difficult than its longitude, for example. This is true with starspot modelling of light curves and of distorted line profiles. One problem, affecting light curve modelling, is needing to know the brightness of an unspotted hemisphere. Another problem, affecting both, is needing to know the inclination of the spin axis. In a few cases, involving modelling of photometry and spectroscopy obtained simultaneously, the resulting spot latitudes seem to be convincing. Examples are Rodonò et al. (1986) and the EI Eri paper mentioned already.

On the other hand, many other spotted stars surely do have spots in their equatorial regions. An obvious example is the many eclipsing binaries with large starspot waves. Because their inclinations are very near $90^{\circ}$, equatorial or nearly equatorial spots are required.

A powerful tool for latitude determination is photometry of eclipsing spotted stars during the secondary eclipse, when the spotted regions are occulted by the smaller companion star. In my opinion, this technique has not been used as much as it should. The secondary eclipse of RS CVn observed at two epochs can be seen in Eaton and Hall (1977, figure 3) and in Eaton and Poe (1985). In both cases the asymmetrical shape indicates the existence of equatorial spots. This matter is elaborated further by Eaton (1990).

Recently a paper by Gray (1989b) demonstrated that active regions, if not actually spots, in giant stars occur preferentially near the equator.

There was a paper by Gray on this subject, latitude dependence, at this meeting. I think it is still true that we do not know what factors determine latitude extent. Is it spectral type or $B-V$, is it rotation speed or Rossby number, is it depth of convection zone, or is it something else

\section{Differential rotation with latitude}

With long-term starspot photometry it is possible to investigate differential rotation as a function of stellar latitude. The results are surprising.

A typical wave in the light curve maintains the same photometric period for a certain interval of time, which can range from months to years but seldom longer than a decade. This indicates that the dark region responsible maintains the same central longitude and a constant rotation period as long as it exists. When a spotted variable is observed continuously for many years, several different spotted regions are seen to appear, exist, and disappear, and typically they have rotation periods which are significantly different from each other. Recent examples of this behavior are V478 Lyr (Hall et al., 1990c), V1817 Cyg = HR 7428 (Hall et al., 1990a), and V1149 Ori = HD 37824 (Hall et al., 1990b). An older example is BY Dra (Oskanyan et al., 1977).

An illustration of the history of starspot rotation periods in RT Lac, covering more than 80 years, is given by Hall and Busby (1990, figure 1) and will not be duplicated here. The horizontal axis is year. The vertical axis is $O-C$, where $O$ is observed time of light minimum (when the spot is facing Earth) and $C$ is time 
computed with an arbitrarily assumed constant rotation period (in this case it is the binary's 5.074-day orbital period). In an $O-C$ curve like this, a positive slope indicates rotation slower than the orbital period, a negative slope indicates rotation faster. In this case the maximum is $0.17 \%$ faster than orbital and the minimum is $0.25 \%$ slower than orbital, for a full range of $\delta P / P=0.4 \%$.

I determined $\delta P / P$ in this way for 84 variable stars of different types, both binary and single. This was largely the same sample used by Hall and Henry (1990), but I have added a few and updated some of the observational material. With the sun included, the sample totals 85 .

The range of spot rotation periods should be a measure of the differential rotation with stellar latitude. Differential rotation in the sun is characterized by the coefficient of a $\sin ^{2} \theta$ term, where $\theta$ is latitude. I'll call this coefficient $k$ and, for the sun, $k=0.186$. Values of $\delta P / P$ for my stars can be converted to values of $k$ if one assumes a latitude range for the starspots, as explained in detail by Hall and Busby (1990, figure 2).

Hall and Henry (1990, Figure 11) had found a relation between $k$ and Rossby number for their sample of stars, in the sense that $k$ decreased when the Rossby number decreased. I would like to show that the relation is just as good if one uses the rotation period instead of the Rossby number (which is defined as the ratio between the rotation period and the convective turnover time). This was the approach used earlier by Rodonò (1986, figure 6) with a much smaller sample of stars.

Linear regression gave the relation

$$
\log k=-2.30( \pm 0.06)+0.85( \pm 0.06) \log \left(P_{\text {rot }}\right),
$$

where $P_{\text {rot }}$ is in days.

It has been suggested that differential rotation might be suppressed or diminished by tidal forces from a companion star. To investigate this matter I did a double regression, i.e., with respect to rotation period and Roche-lobe-fillingfactor, $F$. Because both single stars and contact binaries are in my sample, the filling factors covered the entire range $0<F<1$. The result was

$$
\log k=-2.02( \pm 0.12)+0.79( \pm 0.06) \log \left(P_{\text {rot }}\right)-0.42( \pm 0.16) F
$$

The coefficient of the rotation term is only slightly smaller but there does seem to be a significant correlation with lobe-filling factor, in the sense that stars nearly in contact with their Roche lobes experience diminished differential rotation, about a factor 2.5 less, compared to single stars. This is in disagreement with a conclusion reached earlier by Scharlemann (1982) but it might be consistent with the newer synchronization theory of Tassoul $(1987,1988)$, who shows that synchronization forces are stronger than those based on the tidal theory of Zahn (1977).

When the double regression was repeated, giving $F$ an exponent ranging from 2 to 6 , the resulting coefficient ranged between -0.33 to -0.37 , i.e., within the uncertainty of the value found in equation (2). When the regression was repeated with the ultra-short-period SU UMa binaries omitted from the sample, the result was 


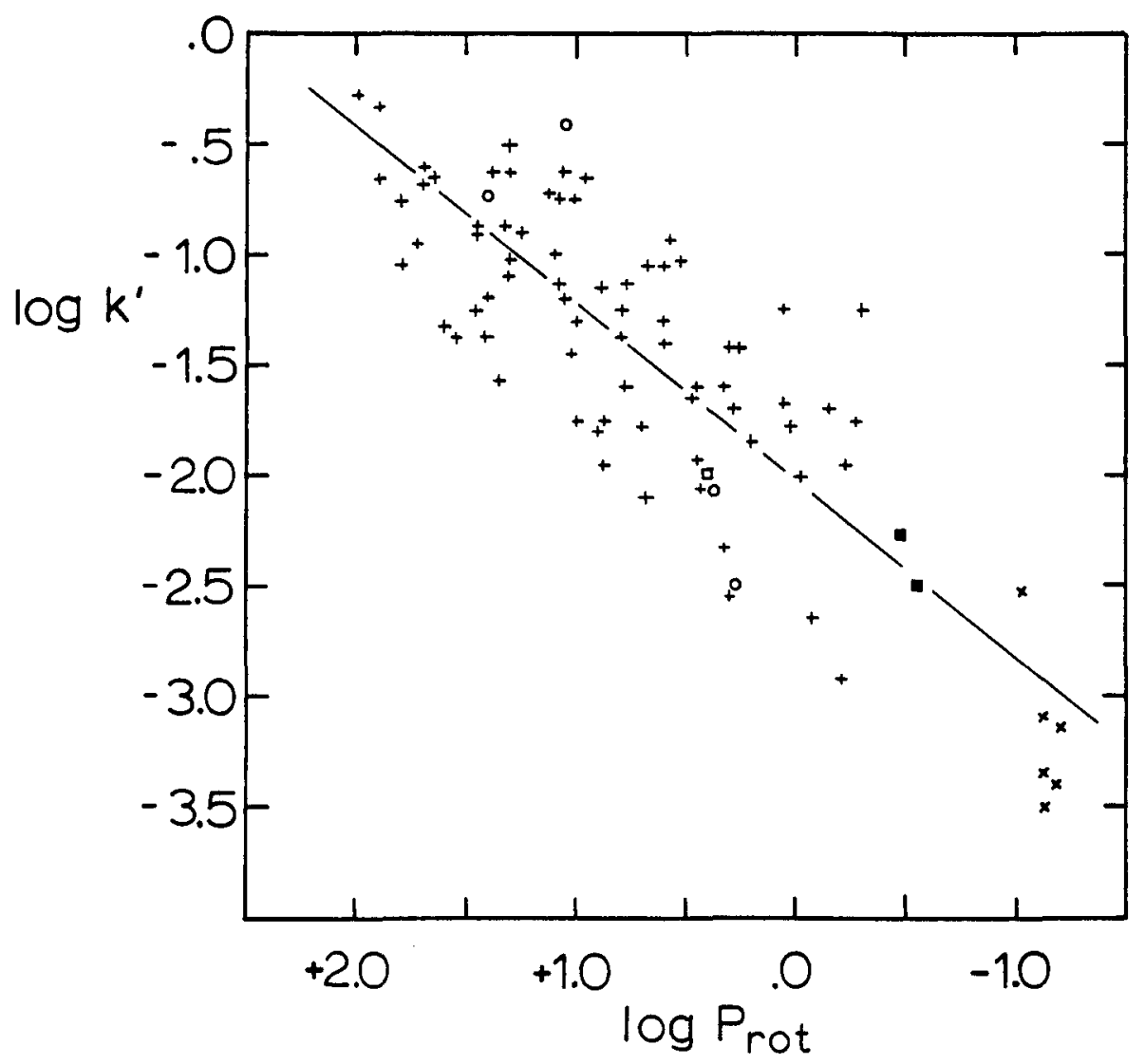

Fig. 7. Differential rotation coefficient $k$, corrected with equation (4), versus rotation period. The straight line thus is equation (2). Different types of stars, both single and in binaries, have different symbols, as explained in the text. Note that those with very short periods are nearly rigid rotators.

$$
\log k=-1.96( \pm 0.12)+0.71( \pm 0.07) \log \left(P_{\text {rot }}\right)-0.38( \pm 0.16) F
$$

Within the uncertainties, this is not significantly different from equation (2) so the observed correlation is not dependent on inclusion of the SU UMa binaries.

To display the results graphically, I have removed the lobe-filling dependence with the relation

$$
\log k^{\prime}=\log k+0.4 F
$$

and then plotted $\log k^{\prime}$ versus $P_{\text {rot }}$ in Figure 7. The slope of the straight line represents the coefficient $0.79 \pm 0.06$. Chromospherically active binaries of the RS CVn 
type or BY Dra type are plusses, the Algol-type binary U Cep is an open square, two W UMa binaries are filled squares, six SU UMa binaries are crosses, and four single stars (the spotted T Tau variable V410 Tau, the chromospherically active giant FK Com, the solar-type star $\epsilon$ Eri, and the Sun) are open circles. Note that differential rotation becomes much less as rotation becomes faster. For the most rapid rotators in my sample, the coefficient $k$ is 300 times smaller than the solar value and 1000 times smaller than the largest value in my sample (for HR 7428). That is very close to rigid-body rotation.

I'm not really sure what the predictions of dynamo theory are concerning differential rotation, but I think most linear dynamo theories predict it should increase as Rossby number decreases or rotation becomes faster. That is definitely not what is seen here. On the other hand, I think non-linear dynamo theories suggest differential rotation should be suppressed by very strong dynamo action, which is what we see here. In any event, I want to emphasize this clear and dramatic observational result, which should be useful in testing predictions of dynamo theories.

Let me clarify that these results pertain only to differential rotation with latitude and say nothing directly about differential rotation with depth down into the convective zone. We can see only the outside of these stars.

\section{Latitude drift during a solar cycle}

We all know that sunspots form at progressively lower latitudes as the 11-year cycle progresses. Do we see the same thing in other stars

Look again at the previously mentioned spot rotation period curve for RT Lac (Hall and Busby 1990, figure 1). If RT Lac had solar-type differential rotation and solar-type latitude drift, we would see spot rotation periods becoming progressively shorter. In this curve the slopes would go gradually from positive to negative. That is not seen; they just jump back and forth.

Another example is V478 Lyr. Its spot rotation period curve during its 9-year photometric history is given by Hall et al. (1990c, figure 5). Again the spot rotation periods jump back and forth with no smooth trend.

Yet another example is HK Lac, which has a 14-year continuous photometric history (Olah et al., 1990). In this case four long-lived spots have been followed and a fifth spot has appeared recently. Each one had a constant rotation period during its lifetime and in this case the four rotation periods were the same, again no indication of a gradual change with time.

To the best of my knowledge, the phenomenon of latitude drift in spotted stars other than the sun has not been convincingly demonstrated. Perhaps it does exist but we have not examined observations which cover a complete cycle. Or perhaps solar-type latitude drift does not exist in heavily spotted stars. 


\section{Sector structure and preferential longitudes}

There has been some recent observational evidence that starspot formation is restricted to two regions of stellar longitude, $180^{\circ}$ apart. See the discussion in Hall (1987, section 5.3). That would be an indication of sector structure, namely, four sectors, which are active, inactive, active, inactive.

There has also been recent observational evidence that these longitudes, if defined with respect to the line of centers in a binary system, remain stationary. That effect would be termed preferential longitudes.

One of the first examples was SV Cam. Zeilik et al. (1987, figure 1) showed a Mercator projection: stellar latitude and stellar longitude. Each circle represented a starspot region observed some time during the 45-year photometric history of SV Cam, with the diameter of each circle proportional to the starspot's radius. One sees a tendency for spots to occur at phases 0.25 and 0.75 , i.e., at the quadratures. Zeilik and collaborators have similar results for other (mostly short-period RS CVn-type) binaries, and Zeilik himself reviewed starspot activity on these systems at this meeting.

Another example is UX Ari. Hall and Strassmeier (1988, figure 1) show four light curves obtained with an automatic telescope in Arizona for four consecutive years. Notice that light minimum of the starspot wave remains stationary at phase 0.95 , i.e., very near one of the conjunctions. This would place the spot on the hemisphere opposite the other star. If we add earlier light curves, discussed by Wacker and Guinan (1987), we see that the spot in UX Ari has remained anchored at phase 0.95 for 7 years.

Another example may be $\zeta$ And, a chromospherically active binary known to show a relatively large ellipticity effect. In the light curve obtained between 1983 and 1986 with an automatic telescope in Arizona (Strassmeier et al., 1989, figure 52 ) the amplitude of the ellipticity effect was about $0 .^{m} 06$ in $V$. At two much earlier epochs the amplitude appeared to be more than twice as large. The ellipticity effect itself, of course, should not change amplitude. Thus we may have had spots during the two earlier epochs, in both cases occurring at the two conjunctions, or we may have had spots during the later epoch, occurring at the two quadratures. Analysis by Hall (1990a) indicates the first of those two possibilities would be the more likely.

Similar results were found with V478 Lyr, as discussed by Hall et al. (1990c). In that case the preferential longitudes, at the moments of spot origin, were 0.0 or 0.5 , i.e., at the conjunctions.

A final example is HK Lac, as discussed by Olah et al. (1990). The four starspots have had relatively long lifetimes, about 7 years each, and each one has migrated in phase away from its point of origin. The longitude at the moment of origin, however, seems to have been the same for all four, namely, phase 0.6.

These results are quite interesting and potentially useful, because some dynamo theories can make a statement about sector structure. An important question, however, is whether it is physically reasonable that tidal forces from a companion star can influence the orientation of sectors, i.e., induce preferential longitudes. 


\section{Starspot lifetimes}

For some time photometry of starspot waves has indicated that these large spots live very long, many years, a few longer than a decade. At one time this was puzzling, because differential rotation at the solar rate would disrupt such large spots within months. People asked what mysterious force held the large spots together.

It seems there is no mysterious force. The greatly diminished differential rotation, discussed in Section IV, makes calculated disruption times correspondingly longer. Hall and Busby (1990) demonstrated that no large starspot has been observed with a lifetime longer than the calculated disruption time. The details of that demonstration are as follow.

Calculated disruption times depend mostly on 3 factors: the spot radius, the differential rotation coefficient, and the rotation period. There is a weaker dependence on the latitude of the spot. Spot radii can be estimated from the amplitude of the starspot wave (Hall and Busby 1990, figure 3). I have already discussed how one estimates the differential rotation coefficient for a spotted star. And the rotation period itself is readily available. The calculated disruption time for a given spot on a given star can be gotten from Hall and Busby (1990, figure 5 and equation 5).

Results for 40 spots on 17 different stars were shown by Hall and Busby (1990, figure 7). They found that for relatively large spots, greater than about $20^{\circ}$ in radius, observed lifetimes $\left(t_{O}\right)$ are equal to their calculated disruption times $\left(t_{C}\right)$. The equality was quite close, because the rms scatter from the $t_{O}=t_{C}$ line was only 0.3 in the log, only a factor 2 .

This agreement $\left(t_{O}=t_{C}\right)$ cannot be the result of a triviality, because two of the three parameters which determine $t_{C}$ cover enormous ranges and so does the observed lifetime itself. Rotation periods in the Hall-Busby sample ranged from 0.6 days to 385 days $(600 \mathrm{X}), k$ ranged from 0.0006 to $0.06(100 \mathrm{X})$, and $t_{O}$ ranged from 0.3 yrs to $30 \mathrm{yrs}(100 \mathrm{X})$.

Hall and Busby (1990, figure 6) found a different relation for the smaller spots, smaller than about $20^{\circ}$ in radius. For them, in general, $t_{O}<t_{C}$. Moreover, when they plotted $t_{O}$ versus spot radius (but on a scale linear with radius squared, i.e., spotted area), they saw that the relatively small spots have lifetimes more or less proportional to their areas. Recall that, for spots on the sun, there is also a rough area-versus-lifetime proportionality. A next step in this investigation, not done by them, would be to consider absolute area, rather than angular area, of each spot. That might decrease the scatter they found in their relation.

The remarkably close agreement between $t_{O}$ and $t_{C}$ (for the large spots) indicates that lifetimes of large spots are in fact limited by shear. This observation is consistent with either of two scenarios: (1) a large spot or active region originates in a deep layer which may be rigidly rotating, is magnetically disconnected after awhile, and is disrupted by differential rotation characterizing the surface, or (2) a large spot or active region is not disconnected magnetically but the deeper layers 
from which it originated approximately mirror the same differential rotation law which applies to the surface layers.

\section{Magnetic cycles}

We all know the sun has a magnetic cycle. It is also rather well established that some other stars have magnetic cycles too, probably physically analagous. There was a good review about five years ago by Baliunas and Vaughan (1985).

Table 3. Types of stars displaying magnetic cycles

1. RS CVn binaries
2. BY Dra variables
3. Algol-type binaries
4. W UMa binaries
5. the sun
6. solar-type dwarfs
7. flare stars
8. U Gem binaries
9. SU UMa binaries
10. old novas
11. recurrent novas
12. low-mass $\mathbf{x}$-ray binaries
13. RR Lyrae variables
14. Cepheids
15. RV Tauri variables

Table 4. Signatures of magnetic cycles

1. the sunspot cycle - sunspot numbers
2. the sunspot cycle - latitude drift
3. long-term changes in Ca II $\mathrm{H} \& \mathrm{~K}$ emission
4. long-term changes in mean brightness
5. cyclic changes in orbital period
6. frequency of flaring in flare stars
7. variable frequency of outbursts in dwarf novas
8. frequency of recurrent nova outbursts
9. the high-low states of Her $\mathrm{X}-1$ and HZ Her
10. cyclic changes in the Blazhko effect in RR Lyrae stars
11. variable magnetic fields in RR Lyrae stars
12. variable pulsation periods in RR Lyrae stars
13. variable pulsation periods in Cepheids
14. variable pulsation periods in RV Tauri variables

For my contribution, I want to explain that magnetic cycles may occur on more types of stars than have been considered heretofore. In demonstrating this I want to explain also that magnetic cycles have more signatures than have been considered heretofore. Finding observational evidence of cycles on many very different types of objects should be more useful for dynamo theory than if we look narrowly only at solar-type stars.

It may turn out that I have cast my net too wide and that the cycles in some of my suggested candidates are not actually magnetic in origin. On the other hand, many of the types of objects I consider display cyclic phenomena which until now 
have no other explanation. Thus, for them, magnetic cycles represent the best, i.e., the only, explanation.

Table 3 lists 15 types of objects which clearly do, probably do, or perhaps do have magnetic cycles. The RV Tauri variables have been added to the list compiled earlier by Hall (1990b) in his review of this same subject. A pertinent reference would be Percy et al. (1989).

Table 4 lists the different observational signatures of magnetic cycles. Hall (1990b) explained in detail how possible magnetic cycles might be expected to produce these various observational manifestations. There is not much to add except that the earlier short note by Bianchini (1988) has been followed by a much more complete discussion (Bianchini, 1990), in which he considers the situation with about half of the 15 types of objects listed in Table 3.

Hall (1990b, table 1) concluded with a summary of different types of objects which display cyclic behavior. For each he gave the median cycle length and, for those which undergo changes in a period, the typical size of those changes. Noting that the cycle lengths for all fall in the range $10 \mathrm{yrs}$ to $100 \mathrm{yrs}$, he suggested that this similarity in timescale is, by itself, suggestive that a common mechanism is at work.

I conclude with an exploratory plausibility argument. Various dynamo theories have the capability of predicting a magnetic cycle. One of them is based on the time which the mean magnetic field needs to diffuse over a characteristic distance, for example, the depth of the convective zone. It predicts that cycle length should depend on the convective turnover time, the size of a convective cell, and the thickness of the convective zone. An equation was given by Hall (1990b, equation 6). If one considers four rather different types of stars (a low-mass main-sequence dwarf, the sun, a middle-G subgiant, and an early- $K$ giant) and uses rough estimates for the three parameters mentioned above, the predicted cycle lengths cover a relatively narrow range, from about 10 years to 100 years (Hall 1990b, table 2). This is the same range found from the observations.

Work in this area needs to be continued. The observational evidence needs to be made stronger, for some of the types of objects on my list, and the comparison with theory needs to be done more rigorously than I have done. But the approach from both directions, observation and theory, promises to answer a lot of important questions, both observational and theoretical.

\section{References}

Baliunas, S.L., Vaughan, A.H.: 1985, Ann. Rev. Astr. Astrophys. 23, 379

Bianchini, A.: 1988, I.B.V.S. 3136

Bianchini, A.: 1990, Astron. J. 99, 1941

Catalano, S., Frisina, A., Rodonò, M.: 1980, I.A.U. Symposium 88, 405

Doyle, J.G., Butler, C.J., Morrison, L.V., Gibbs, P.: 1988, Astron. Astrophys. 192, 275

Eaton, J.A.. 1990, in Surface Inhomogeneities in Late-Type Stars, eds P.B. Byrne and

D.F. Mullan (Berlin: Springer-Verlag), in press

Eaton, J.A., Hall, D.S.: 1977, Astrophys. J. 227, 907 
Eaton, J.A., Poe, C.H.: 1985, B.A.A.S. 16, 914

Gilliland, R.: 1985, Astrophys. J. 299, 286

Gray, D.F.: 1988, Lectures on Spectral Line Analysis of F, $G$, and $K$ Stars, Arva, Ontario Gray, D.F.: 1989a, Astrophys. J. 347, 1021

Gray, D.F.: 1989b, Publ. Astron. Soc. Pac. 101, 695

Hall, D.S.: 1987, Publ. Astr. Inst. Czechoslovakia 70, 77

Hall, D.S.: 1989, I.A.U. Colloquium 107, 219.

Hall, D.S.: 1990a, Astron. J. 100, 554

Hall, D.S.: 1990b, in Active Close Binaries, ed. C. Ibanoglu (Dordrecht: Kluwer), p. 95.

Hall, D.S., Busby, M.R.: 1990, in Active Close Binaries, ed. C. Ibanoglu (Dordrecht: Kluwer), p. 377.

Hall, D.S., Gessner, S.E., Lines, H.C., Lines, R.D.: 1990a, Astron. J. 100, 2017

Hall, D.S., Henry, G.W.: 1990, in Active Close Binaries, ed. C. Ibanoglu (Dordrecht: Kluwer), p. 287.

Hall, D.S., Henry, G.W., Barksdale, W.S.: 1990b, in preparation.

Hall, D.S., Henry, G.W., Sowell, J.R.: 1990c, Astron. J. 89, 396

Hall, D.S., Strassmeier, K.G.: 1988, in Automatic Small Telescopes, eds. D.S. Hayes and

R.M. Genet (Mesa: Fairborn Press), p. 71.

Olah, K., Hall, D.S., Henry, G.W.: 1990, in preparation.

Oskanyan, V.S., Evans, D.S., Lacy, C., McMillan, R.S.: 1977, Astrophys. J. 214, 430

Percy, J.R., Sasselov, D.D., Alfred, A., Scott, G.: 1989, B.A.A.S. 21, 111

Rodonò, M:. 1986, in Cool Stars, Stellar Systems, and the Sun, eds. M. Zeilik and D.M. Gibson (Berlin: Springer-Verlag), p. 475.

Rodonò, M. et al. [22 authors]: 1986, Astron. Astrophys. 165, 135

Scharlemann, E.T.: 1982, Astrophys. J. 253, 298

Strassmeier, K.G. 1989, in Remote Access Small Telescopes, eds. D.S. Hayes and

R.M. Genet (Mesa: Fairborn Press), p. 223.

Strassmeier, K.G.: 1990, Astrophys. J. 348, 682

Strassmeier, K.G., Hall, D.S.: 1988, Astrophys. J. Suppl. 67, 453

Strassmeier, K.G., Hall, D.S., Barksdale, W.S., Jusick, A.T., Henry, G.W.: 1990, Astrophys. J. 350, 367

Strassmeier, K.G., Hall, D.S., Boyd, L.J., Genet, R.M.: 1989, Astrophys. J. Suppl. 69, 141

Strassmeier, K.G., Hall, D.S., Zeilik, M., Nelson, E., Eker, Z., Fekel, F.C.: 1988, Astron. Astrophys. Suppl. 72, 291

Tassoul, J.P.: 1987, Astrophys. J. 322, 857

Tassoul, J.P.: 1988, Astrophys. J. 324, L71

Wacker, S., Guinan, E.F.: 1987, I.B.V.S. 3018

Zahn, J.P.: 1977, Astron. Astrophys. 57, 383

Zeilik, M., De Blasi, C., Rhodes, M., Budding, E.: 1987, in Cool Stars, Stellar Systems, and the Sun, eds. J.L. Linsky and R.E. Stencel (Berlin: Springer-Verlag), p. 503. 